\title{
The impact of comorbidity on survival after hemorrhagic stroke among dialysis patients: a nationwide population-based study
}

Chun-Yu Lin ${ }^{1,2}$, Chih-Chiang Chien ${ }^{3,4}$, Hung-An Chen ${ }^{1,5}$, Fu-Mei Su', Jhi-Joung Wang ${ }^{7}$, Che-Chuan Wang ${ }^{8,9,10}$, Chin-Chen $\mathrm{Chu}^{11}$ and Yeong-Jang Lin ${ }^{1 *}$

\begin{abstract}
Background: This study was aimed at determining the outcome and examining the association between comorbidities and mortality after intracerebral hemorrhage in chronic dialysis patients.

Methods: We used the Taiwan National Health Insurance Research Database and enrolled patients who underwent maintenance dialysis between 2000 and 2007. Annual incidence of intracerebral hemorrhage in patients receiving dialysis from 2000 to 2007 was determined. To identify predictors of hemorrhagic stroke, we used logistic regression model to estimate the relative ratio of factors for intracerebral hemorrhage in the most recent cohort (2007). The cumulative survival rate and comorbid conditions associated with mortality after intracerebral hemorrhage among all dialysis patients between 2000 and 2007 was calculated using the Kaplan-Meier method and Cox regression analysis.

Results: We identified 57,261 patients on maintenance dialysis in the cohort of 2007, and 340 patients had history of intracerebral hemorrhage among them. Hypertension was the most common comorbidity of dialysis patients. The incidence rate of intracerebral hemorrhage among dialysis patients was about $0.6 \%$. Adjusted logistic regression model showed that male gender, middle age (45-64 years), hypertension, and previous history of stroke were the independent predictors for the occurrence of intracerebral hemorrhage among chronic dialysis patients. 1,939 dialysis patients with development of intracerebral hemorrhage in the analysis period from 2000 to 2007 were identified. In-hospital mortality was high (36.15\%) following intracerebral hemorrhage. They were followed up after intracerebral hemorrhage for a mean time of 41.56 months. Adjusted Cox regression analyses demonstrated that the factors independently associated with mortality after intracerebral hemorrhage among dialysis patients included diabetes mellitus, malignancy and a history of prior stroke.
\end{abstract}

Conclusions: Dialysis patients who have history of prior stroke, diabetes and malignancy have worse survival than patients without these comorbidities. Attention must focus on providing optimal medical care after hemorrhagic stroke for these target groups to reduce mortality.

Keywords: Intracerebral hemorrhage, Dialysis, Comorbidity, Mortality

\footnotetext{
* Correspondence: linyeongjang@yahoo.com.tw

${ }^{1}$ Division of Allergy-Immunology-Rheumatology, Department of Internal

Medicine, Chi Mei Medical Center, Tainan, Taiwan

Full list of author information is available at the end of the article
} 


\section{Background}

Cerebrovascular disease is a common cause of morbidity and mortality in patients with end-stage renal disease (ESRD). Patients with ESRD have advanced atherosclerosis of the cerebral vasculature [1]. Stroke rates for ESRD patients are markedly higher than for the general population [2]. Intracerebral hemorrhage (ICH) is a type of stroke that involves bleeding within the brain tissue itself. It is well known that a bleeding tendency is a major concern among ESRD patients. In addition, the routine administration of heparin during hemodialysis may increase the risk of bleeding. ICH is more common in dialysis patients than in the general population [2], and is a devastating and life-threatening condition that requires urgent intervention. The onset of $\mathrm{ICH}$ is usually sudden and, unlike ischemic stroke symptoms, ICH symptoms tend to appear without warning. $\mathrm{ICH}$ in dialysis patients has a poor prognosis and a very high mortality rate, despite advances in diagnosis and treatment $[3,4]$. It is important to identify dialysis patients at risk for developing $\mathrm{ICH}$ and the prognostic factors after $\mathrm{ICH}$.

$\mathrm{ICH}$ is more common among Taiwanese than Caucasians [5]. The incidence and prevalence rates of ESRD are also high in Taiwan [6]. However, little is known about the annual incidence of $\mathrm{ICH}$ in the Taiwanese dialysis population. There are also few nationwide studies analyzing the predictors of $\mathrm{ICH}$ and the effect of comorbidities on survival following $\mathrm{ICH}$ in patients on chronic dialysis. In the study, we utilized a large data set from the National Health Insurance Research Database (NHIRD) to identify predicting factors for intracerebral hemorrhage in dialysis patients as the primary objective. The secondary objective was to report the clinical outcomes and identify the independent predictors of $\mathrm{ICH}$ mortality in these patients.

\section{Methods \\ Data source}

Data for this cohort study was obtained from the NHIRD (http://nhird.nhri.org.tw), which was released for research purposes by the Taiwan National Health Research Institute. The NHIRD, which includes nearly all (99\%) of the inpatient and outpatient medical benefit claims for the Taiwanese population of 23 million people, is one of the largest and most comprehensive databases in the world and has been used extensively in various studies [7]. The dataset was released with de-identified secondary data for public research purposes. All types of personal identification on files connected with the present study were scrambled using surrogate identification numbers to secure patient privacy. The study was exempt for the need of written informed patient consent by the Institutional Review Board of Chi-Mei medical center (1008-002) and the Bureau of National Health
Insurance (NHRI-NHIRD-99182). We used the NHIRD to collect the ambulatory care claims, all inpatient claims, and the updated registry for beneficiaries from 2000 to 2007 for this study. All datasets can be interlinked through each individual's unique personal identification number.

\section{Patient selection and definition}

We enrolled ESRD patients in the NHIRD who underwent maintenance dialysis from January 1, 2000 to December 31, 2007. Patients with ESRD are eligible for any type of renal replacement therapy free of charge and without co-payments in Taiwan. Patients on maintenance dialysis were defined as having undergone dialysis longer than 90 days. Patients who had undergone renal transplantation before the initiation of dialysis were excluded. We linked study subjects to the inpatient claims data to identify newly diagnosed ICH (ICD-9-CM code 431).

\section{Ascertaining the demographic data and comorbidities}

Data on age, gender, initial modality, and baseline comorbidities were recorded for each dialysis patient. The baseline comorbidities included diabetes mellitus, hypertension, heart failure, coronary artery disease, old stroke, peripheral arterial disease, chronic lung disease, chronic liver disease, and cancer. Selected comorbidities were ascertained based on 1 in-patient claim or 3 out-patient claims at the start of dialysis or during the follow-up. The ICD-9-CM codes used to define clinical conditions are shown in Additional file 1.

\section{Statistical analyses}

We assessed the annual incidence of ICH among dialysis patients from 2000 to 2007. For each annual cohort, we tabulated all characteristics by presence versus absence of previous $\mathrm{ICH}$, with continuous variables using means and standard deviation and categorical variables using counts and percentages. Because our selected subjects were prevalent dialysis patients, which were not known the time of initiation of dialysis, we used the most recent cohort, for the year 2007, to identify the predictors of $\mathrm{ICH}$ and used logistic regression models to estimate the relative ratio for each factor. The cumulative survival rate after developing $\mathrm{ICH}$ was calculated using the Kaplan-Meier method. Hazard ratios for each factor were calculated using Cox regression analysis to determine significant predictors for mortality among all dialysis patients with occurrence of ICH between 2000 and 2007. If not addressed, p-values less than 0.05 were considered statistically significant. Data analysis was performed using SPSS for Windows, version 17.0 (SPSS Inc., Illinois, USA). 


\section{Results}

\section{Baseline demographic and clinical data}

The patients on maintenance dialysis suffering from $\mathrm{ICH}$ accounted for less than $1 \%$ of the total dialysis cohort. The majority of patients used hemodialysis as their first modality of renal replacement therapy, but the rate of utilization of peritoneal dialysis as primary treatment for ESRD increased in the analysis period. The prevalence of comorbid conditions in the ESRD population without ICH was substantial: hypertension was the most common, followed by diabetes mellitus, chronic liver disease, coronary artery disease, congestive heart failure, prior stroke, chronic lung disease, cancer, and peripheral artery disease, based on the year 2007 cohort. The frequency of comorbidities among dialysis patients with $\mathrm{ICH}$ was somewhat different from those without $\mathrm{ICH}$; i.e., prior stroke was the third most common disease. Moreover, the prevalence of diabetes, hypertension and previous history of stroke among dialysis patients suffering from ICH was even greater. The percentage of patients suffering from $\mathrm{ICH}$ in the peritoneal dialysis group was less than in the hemodialysis group (Table 1).

\section{Incidence rate and predictors of ICH in dialysis patients}

The incidence rate of $\mathrm{ICH}$ in the dialysis population was about $0.6 \% .57,261$ patients on maintenance dialysis were identified in the most recent and largest cohort of 2007. Results of adjusted logistic regression analysis showed that male gender, middle age (45-64 years at baseline), hypertension, and previous history of stroke were the independent predictors for $\mathrm{ICH}$ among chronic dialysis patients. ESRD patients on hemodialysis tended to have a higher incidence of $\mathrm{ICH}$ than those on peritoneal dialysis; however, there was no statistical significance (Table 2).

\section{Predictors for all-cause mortality after ICH in dialysis patients}

1,939 dialysis patients with the occurrence of intracerebral hemorrhage from 2000 to 2007 were identified. The mean of follow-up time after ICH was 41.56 months. Overall, we found a high in-hospital mortality rate (36.15\%) among dialysis patients hospitalized with $\mathrm{ICH}$. In the univariate analysis of baseline data, diabetes mellitus, hypertension, coronary artery disease, previous history of stroke, chronic liver disease, and malignancy were associated with higher mortality following ICH hospitalization. The multivariate Cox regression analysis showed that previous history of stroke, diabetes mellitus and malignancy were independent predictors of mortality after ICH among dialysis patients. The in-hospital mortality rate in patients with a prior history of stroke was almost the same as in patients without a prior history of stoke (36.14\% vs. $36.16 \%)$. Crude overall survival curves after intracerebral hemorrhage among dialysis patients with and without prior stroke were shown in the Figure 1. The two survival curves started to separate at

Table 1 Characteristics of patients on maintenance dialysis in selected years between 2000 and 2007 by presence versus absence of ICH

\begin{tabular}{|c|c|c|c|c|c|c|}
\hline \multirow[t]{2}{*}{ Characteristics } & \multicolumn{2}{|c|}{2000} & \multicolumn{2}{|c|}{2004} & \multicolumn{2}{|c|}{2007} \\
\hline & No ICH $n=34282$ & $\mathrm{ICH} n=230$ & No ICH $n=47555$ & $\mathrm{ICH} n=289$ & No ICH $n=56921$ & $\mathrm{ICH} n=340$ \\
\hline \multicolumn{7}{|c|}{ No. (\%) } \\
\hline Age (mean $\pm S D)$, y & $57.3 \pm 14.5$ & $58.9 \pm 12$ & $59.1 \pm 14.1$ & $58.6 \pm 12.5$ & $60.7 \pm 13.9$ & $60.7 \pm 11.9$ \\
\hline Female & $18303(53.4)$ & $107(46.5)$ & $25598(53.8)$ & $120(41.5)$ & $30186(53.0)$ & $155(45.6)$ \\
\hline \multicolumn{7}{|l|}{ Dialysis modality } \\
\hline Hemodialysis & $32340(94.3)$ & $224(97.4)$ & $44408(93.4)$ & $279(96.5)$ & $52569(92.4)$ & $320(94.1)$ \\
\hline Peritoneal dialysis & $1942(5.7)$ & $6(2.6)$ & $2147(6.6)$ & $10(3.5)$ & $4352(7.6)$ & $20(5.9)$ \\
\hline ICH in-hospital days & & $11.2( \pm 15.9)$ & & $10.9( \pm 11.3)$ & & $13.2( \pm 15.2)$ \\
\hline \multicolumn{7}{|l|}{ Comorbid conditions } \\
\hline Hypertension & $17192(50.1)$ & $143(62.2)$ & $25044(52.7)$ & $170(58.8)$ & $33072(58.1)$ & $238(70.0)$ \\
\hline Diabetes & $11356(33.1)$ & $101(43.9)$ & $15897(33.4)$ & $118(40.8)$ & $21559(37.9)$ & $166(48.8)$ \\
\hline Chronic liver disease & 7528 (21.9) & $26(11.3)$ & $11902(25.0)$ & $59(20.4)$ & $14203(24.9)$ & $91(26.8)$ \\
\hline Coronary artery disease & $6252(18.3)$ & $48(20.9)$ & $9290(19.6)$ & $67(23.2)$ & $12355(21.7)$ & $88(25.9)$ \\
\hline Heart failure & $3795(11.1)$ & $33(14.3)$ & $5734(12.1)$ & $34(11.8)$ & 7300 (12.8) & $46(13.5)$ \\
\hline Prior stroke & $3153(9.3)$ & $44(19.1)$ & $4633(9.8)$ & $63(21.8)$ & $5815(10.3)$ & $91(26.8)$ \\
\hline Chronic lung disease & $3021(8.8)$ & $19(8.3)$ & $3878(8.2)$ & $24(8.3)$ & $4209(7.4)$ & $21(6.2)$ \\
\hline Peripheral artery disease & $1858(5.4)$ & $11(4.8)$ & $1621(3.4)$ & $9(3.1)$ & $2803(4.9)$ & $27(8.7)$ \\
\hline Cancer & $1116(3.2)$ & $2(0.9)$ & $2752(5.8)$ & $13(4.5)$ & $4150(7.3)$ & $21(6.2)$ \\
\hline
\end{tabular}

$\mathrm{ICH}$, Intracerebral hemorrhage. 
Table 2 Logistic regression model to evaluate for predictors of incidence of intracerebral hemorrhage in patients on maintenance dialysis

\begin{tabular}{lcc}
\hline \multicolumn{1}{c}{ Factors } & $\begin{array}{c}\text { Univariate } \\
\text { RR (95\% Cl) }\end{array}$ & $\begin{array}{c}\text { Multivariate } \\
\text { RR (95\% Cl) }\end{array}$ \\
\hline $\begin{array}{l}\text { Sex (Male v Female) } \\
\text { Age }\end{array}$ & $1.35(1.09-1.67)^{*}$ & $1.25(1.01-1.55)^{*}$ \\
$\quad<44$ (Referent) & 1 & 1 \\
$45-64$ & $1.86(1.25-2.77)^{*}$ & $1.56(1.04-2.34)^{*}$ \\
$\geq 65$ & $1.44(0.96-2.17)$ & $1.09(0.71-1.67)$ \\
Dialysis modality (PD v HD) & $0.76(0.48-1.19)$ & $0.81(0.51-1.29)$ \\
Comorbidities & & \\
Diabetic mellitus & $1.57(1.26-1.94)^{*}$ & $1.15(0.91-1.46)$ \\
Hypertension & $1.68(1.33-2.12)^{*}$ & $1.37(1.06-1.77)^{*}$ \\
Heart failure & $1.06(0.78-1.45)$ & $0.90(0.65-1.24)$ \\
Coronary artery disease & $1.26(0.99-1.61)$ & $1.04(0.80-1.35)$ \\
Prior stroke & $3.21(2.52-4.09)^{*}$ & $2.86(2.22-3.69)^{*}$ \\
Peripheral arterial disease & $1.67(1.12-2.47)^{*}$ & $1.41(0.94-2.10)$ \\
Chronic lung disease & $0.82(0.53-1.28)$ & $0.74(0.47-1.16)$ \\
Chronic liver disease & $1.10(0.86-1.40)$ & $1.03(0.81-1.31)$ \\
Cancer & $0.84(0.54-1.30)$ & $0.90(0.57-1.40)$ \\
\hline
\end{tabular}

*significant difference.

$P D$, Peritoneal dialysis; $H D$, Hemodialysis; $C l$, Confidence interval; $R R$, Relative ratio.

about 8 weeks after $\mathrm{ICH}$. The crude survival rates at 1 year and 2 years after ICH differed between dialysis patients with and without a history of prior stroke ( $45.7 \%$ vs. $49.4 \%, 35.5 \%$ vs. $44.1 \%$ respectively) (log rank: $P=0.02$ ) (Figure 1) (Table 3).

\section{Discussion}

This nationwide study was designed to investigate the risk factors of $\mathrm{ICH}$ occurrence and predictors of mortality following $\mathrm{ICH}$ in prevalent dialysis patients using the Taiwan National Health Insurance (NHI) database. We found a high incidence rate of ICH among chronic dialysis patients. Male gender, middle age, hypertension, and history of old stroke were independent factors of incident ICH. Diabetes mellitus and malignancy were significant independent predictors of mortality after ICH. Long-term outcome of ICH survivors was much poorer among the group with a history of prior stroke than among those without a history of prior stroke.

The present findings confirm the high rate of $\mathrm{ICH}$ among dialysis patients compared with the general population reported in a prior study [2]. We also showed that hypertension and male gender were independent predictors of hemorrhagic stroke among patients on chronic dialysis. These observations were consistent with recent study by Yoo et al., who evaluated predictive variables of ICH in dialysis patients using the United States Renal Data System (USRDS) database [8]. Similar results were also observed in previous study by Seliger's [9], in which increased $\mathrm{ICH}$ rate was associated with a raised mean arterial pressure of at least $>10 \mathrm{mmHg}$. Thus, hypertension is a risk factor for $\mathrm{ICH}$ for both the general population and dialysis patients [10]. While higher blood pressure has relevance to incidental $\mathrm{ICH}$, the effect of it on all-cause mortality seems to be different. We did not find an association between hypertension and all-cause mortality after ICH in patients undergoing chronic

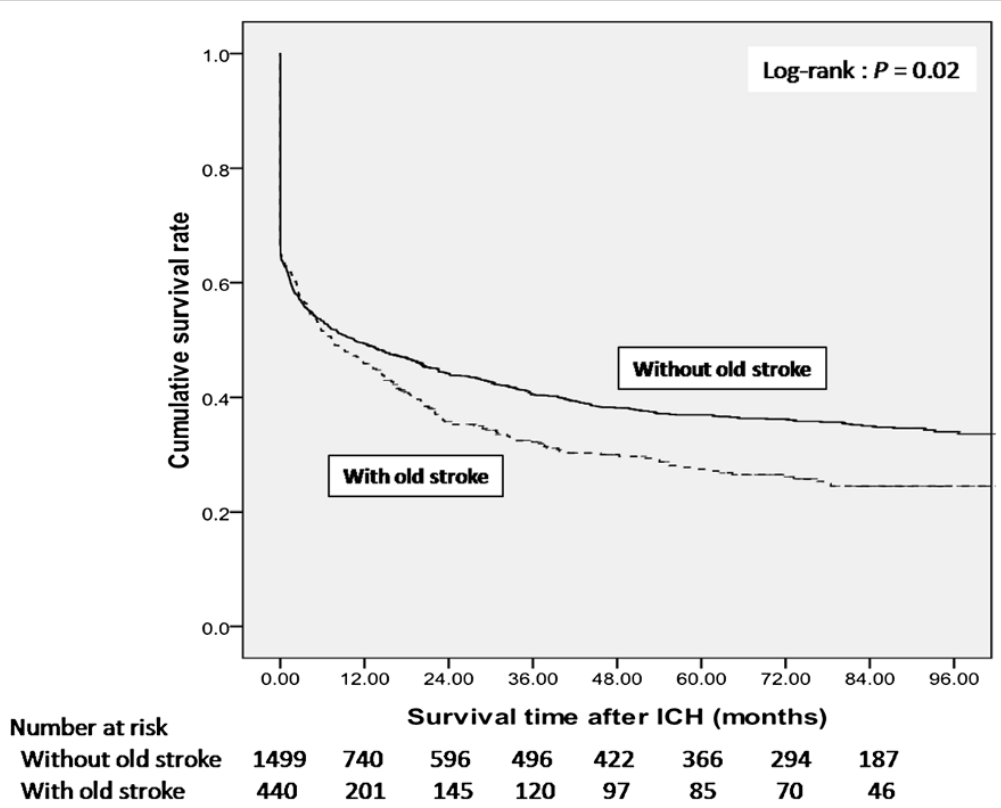

Figure 1 Crude overall survival curves after intracerebral hemorrhage among dialysis patients stratified as being with and without prior stroke. 
Table 3 Cox regression model to evaluate for predictors of all-cause mortality after intracerebral hemorrhage in dialysis patients

\begin{tabular}{|c|c|c|}
\hline Factors & $\begin{array}{c}\text { Univariate } \\
\text { analysis } \\
\text { HR }(95 \% \mathrm{Cl})\end{array}$ & $\begin{array}{c}\text { Multivariate } \\
\text { analysis } \\
\text { HR }(95 \% \mathrm{Cl}) \\
\end{array}$ \\
\hline Sex (male $\vee$ female) & $1.07(0.96-1.20)$ & $1.08(0.96-1.20)$ \\
\hline \multicolumn{3}{|l|}{ Age } \\
\hline$<45$ & 1 & 1 \\
\hline $45-64$ & $1.05(0.86-1.27)$ & $1.01(0.82-1.23)$ \\
\hline$\geq 65$ & $1.20(0.98-1.47)$ & $1.13(0.91-1.39)$ \\
\hline Dialysis modality (PD v HD) & $0.97(0.72-1.32)$ & $1.02(0.75-1.39)$ \\
\hline \multicolumn{3}{|l|}{ Comorbidities } \\
\hline $\begin{array}{l}\text { Diabetic mellitus } \\
\text { (yes } \vee \text { no) }\end{array}$ & $1.15(1.03-1.29)^{*}$ & $1.14(1.01-1.28)^{*}$ \\
\hline Hypertension (yes v no) & $1.06(0.94-1.18)$ & $0.95(0.83-1.08)$ \\
\hline Heart failure (yes v no) & $0.97(0.82-1.15)$ & $0.93(0.78-1.11)$ \\
\hline $\begin{array}{l}\text { Coronary artery disease } \\
\text { (yes } \vee \text { no) }\end{array}$ & $1.15(1.01-1.31)^{*}$ & $1.09(0.95-1.26)$ \\
\hline Prior stroke (yes v no) & $1.20(1.05-1.36)^{*}$ & $1.17(1.03-1.34)^{*}$ \\
\hline $\begin{array}{l}\text { Peripheral arterial disease } \\
\text { (yes } \vee \text { no) }\end{array}$ & $1.11(0.87-1.43)$ & $1.05(0.82-1.35)$ \\
\hline $\begin{array}{l}\text { Chronic lung disease } \\
\text { (yes } \vee \text { no) }\end{array}$ & $1.07(0.87-1.32)$ & $1.01(0.82-1.26)$ \\
\hline $\begin{array}{l}\text { Chronic liver disease } \\
\text { (yes } \vee \text { no) }\end{array}$ & $1.17(1.02-1.33)^{*}$ & $1.12(0.98-1.28)$ \\
\hline Cancer (yes v no) & $1.79(1.39-2.31)^{*}$ & $1.74(1.34-2.25)^{*}$ \\
\hline
\end{tabular}

*significant difference.

$P D$, Peritoneal dialysis; $H D$, Hemodialysis; $H R$, Hazard ratio; $C l$, Confidence interval.

dialysis. Our finding that a history of hypertension is not an independent predictor of death among chronic dialysis patients is in agreement with the work of Tetri and colleagues, who investigated the factors associated with outcome after ICH in the general population [11]. However, the observation of a null association between elevated blood pressure and poor outcome of dialysis patients should be interpreted with some caution. We did not differentiate patients using antihypertensive medications from those without treatment in this analysis, and it seems probable that the effect of hypertension is confounded by the use of these drugs.

This study found that previously diagnosed diabetes predicted mortality. Diabetes was also demonstrated to be an independent determinant of death in the general population suffering from ICH [12]. The exact reason why diabetes is associated with the risk of mortality following ICH remains unclear. The effect of DM on survival may be explained by the severity of hemorrhagic events. Diabetes induces structural and functional changes in the microvasculature that lead to fibrinoid necrosis of blood vessel walls and microaneurysm formation. It is possible that these changes in vessel walls make diabetic patients more likely to develop larger sized hematomas than non-diabetics. There is evidence showing that patients with an elevated glucose level due to diabetes were associated with hemorrhages of a larger size compared to patients with a normal glucose level [13], and that the volume of ICH was a strong predictor of mortality after primary ICH $[14,15]$. In addition, patients with uncontrolled diabetes and a high glucose level may also have a higher risk of infectious or cardiovascular complications during hospitalization.

Moreover, we found that a previous medical history of stroke is strongly associated with both a higher incidence of $\mathrm{ICH}$ and higher mortality risk following $\mathrm{ICH}$ among dialysis patients. The risk of $\mathrm{ICH}$ was reported to increase several times after ischemic infarct in the general population [16,17]. It is well established that dialysis patients have a much higher incidence of cerebral ischemic events. Therefore, it is prudent to avoid overanticoagulation in these patients and to monitor them more closely when providing antithrombotic drugs to prevent recurrent ischemic infarction. A complex series of pathophysiological events are initiated in the brain after ischemic injury. It has been reported that matrix metalloproteinases-2 (MMP-2), MMP-3 and MMP-9, which are capable of digesting basal lamina and extracellular matrix components and lead to subsequent blood brain barrier breakdown and hemorrhagic transformation, were abnormally upregulated in the ischemic region and peri-infarct area [18-20]. Increased expression of MMP-9 of the brain tissue was also observed in patients suffering from hemorrhagic stroke [20]. Moreover, the increased activities of inducible matrix proteases may sustain for several months or years after brain injury [21]. It is possible that physiologic and pathologic responses to stroke cause chronic damage to vessel wall components and pose a sustained risk of subsequent $\mathrm{ICH}$ among dialysis patients.

The present study showed the in-hospital mortality rate among dialysis patients hospitalized with $\mathrm{ICH}$ was $36.15 \%$. This result correlated with previous reports from other Asian countries. In a study from Korea, the 30-day mortality rate among 102 ESRD cases of spontaneous ICH was 53.9\% [22]. Another study from Japan using registered data showed that the death rate of dialysis patients after cerebral hemorrhage was $27.9 \%$ [23]. A recent study from Taiwan reported that the mortality rate within 30 days after spontaneous $\mathrm{ICH}$ in the general population was $19.8 \%$ [24]. Thus, the outcome of ICH is poor among patients on chronic dialysis, and can be worse than in the general population.

Whether gender has an impact on survival after stroke remains controversial. It has been suggested that estrogen may exert neuroprotective effects after brain injury by way of stimulating insulin-like growth factor-I 
synthesis, lessening inflammatory processes, scavenging free radicals, and stimulation of intrinsic anti-apoptotic pathways $[25,26]$. A recent study demonstrated a significant survival advantage for females over males over 3year period after primary ICH [27]. Another study in Denmark also revealed a female superiority in survival time after stroke [28]. However, we did not find such an association between female gender and lower all-cause mortality rates after hemorrhagic stroke among chronic dialysis patients.

Peritoneal dialysis (PD) was claimed to have some benefits, such as minimal anticoagulation and improved cardiovascular or intracranial stability as a consequence of smaller changes in serum osmolality. There is also evidence showing that intracranial pressure increased significantly in patients receiving conventional intermittent hemodialysis [29]. So patients on PD were expected to experience fewer acute cerebral events compared with those on hemodialysis (HD), and some authors suggest a survival advantage with the use of PD instead of HD for chronic dialysis patients with cerebral hemorrhage $[30,31]$. In the USRDS study by Yoo mentioned above, use of peritoneal dialysis as long-term renal replacement therapy was associated with lower risk of $\mathrm{ICH}$ compared to use of hemodialysis [8]. However, there is a contradictory result. A recent work showed an even worse prognosis for patients receiving PD after ICH [4]. Our analysis did not support the association of PD as a dialysis modality with less incidental ICH or decreased mortality following $\mathrm{ICH}$ among chronic dialysis patients.

The strength of our study lies in its large population size, and high likelihood that nearly all relevant patients were collected from the database. The limitations of this study include a lack of laboratory data, and a lack of information about the causes of death, because they were not included in the Taiwan NHI database. We used ICD-9 code 431 to identify cases of $\mathrm{ICH}$, so patients with isolated subarachnoid hemorrhage (ICD-9 code: 430) would not be included in our analysis. We could not affirmatively determine the duration and severity of comorbidities, which may influence outcome of our subjects, in the databases-derived study. We did not have access to detailed information about ICH and dialysis, so the severity and location of the $\mathrm{ICH}$, blood pressure values, and dialysis adequacy cannot be assessed. Surgery was required in patients with large hematoma formation or refractory elevated intracranial pressure. Operation may have an influence on short-term mortality or longterm disability in our study subjects but we did not analyze the proportion of patients receiving surgical treatment after $\mathrm{ICH}$. Moreover, we did not evaluate drug treatment in ESRD population, such as antiplatelet or anticoagulants, which may be associated with incidence of ICH. We also did not differentiate ischemic stroke from hemorrhagic stroke in determining comorbidities. Further large prospective studies with detailed evaluations are needed to confirm our findings.

\section{Conclusion}

In summary, our nationwide study showed that patients on chronic dialysis had a high risk of hemorrhagic stroke. The factors associated with $\mathrm{ICH}$ occurrence and post-hemorrhage survival of these patients were somewhat different from the traditionally reported risk factors for ICH. Dialysis patients that have history of prior stroke, diabetes or malignancy have worse survival than patients without these comorbidities. It is important to identify patients with modifiable risks; this will enable clinicians to provide the patients with preventive strategies and best medical care to achieve better outcomes during the dialysis period.

\section{Additional file}

Additional file 1: ICD-9-CM codes used to identify clinical conditions.

Competing interests

The authors declare that they have no competing interests.

\section{Authors' contributions}

CYL and FMS: Draft the article and provide intellectual content of critical importance to the work described. HAC, JJW, CCW and CCC: Design, analysis and interpretation of data. CCC and YJL: Final approval of the version to be published. All authors read and approved the final manuscript.

\section{Acknowledgements}

This study is based in part on data from the National Health Insurance Research Database provided by the National Health Insurance Administration, Ministry of Health and Welfare and managed by the National Health Research Institutes. The interpretation and conclusions contained herein do not represent those of National Health Insurance Administration, Ministry of Health and Welfare or National Health Research Institutes.

\section{Author details}

'Division of Allergy-Immunology-Rheumatology, Department of Internal Medicine, Chi Mei Medical Center, Tainan, Taiwan. ${ }^{2}$ Division of

Allergy-Immunology-Rheumatology, Department of Internal Medicine, Chi Mei Medical Center, Liouying, Taiwan. ${ }^{3}$ Division of Nephrology, Department of Internal Medicine, Chi Mei Medical Center, Tainan, Taiwan. ${ }^{4}$ Department of Food Nutrition, Chung Hwa University of Medical Technology, Tainan, Taiwan. ${ }^{5}$ Chia Nan University of Pharmacy and Science, Tainan, Taiwan. ${ }^{6}$ Division of Allergy-Immunology-Rheumatology, Department of Internal Medicine, Kaohsiung Chang Gung Memorial Hospital and Chang Gung University College of Medicine, Kaohsiung, Taiwan. ${ }^{7}$ Department of Medical Research, Chi Mei Medical Center, Tainan, Taiwan. ${ }^{8}$ Department of Neurosurgery, Chi Mei Medical Center, Tainan, Taiwan. ${ }^{9}$ Institute of Photonic system, National Chiao Tung University, Tainan, Taiwan. ${ }^{10}$ Department of Child Care, Southern Taiwan University of Science and Technology, Tainan, Taiwan. ${ }^{11}$ Department of Anesthesiology, Chi-Mei Medical Center, Tainan, Taiwan.

Received: 31 July 2014 Accepted: 19 November 2014 Published: 27 November 2014

\section{References}

1. Kennedy R, Case C, Fathi R, Johnson D, Isbel N, Marwick TH: Does renal failure cause an atherosclerotic milieu in patients with end-stage renal disease? Am J Med 2001, 110(3):198-204. 
2. Seliger SL, Gillen DL, Longstreth WT Jr, Kestenbaum B, Stehman-Breen CO: Elevated risk of stroke among patients with end-stage renal disease. Kidney Int 2003, 64(2):603-609.

3. Huang BR, Liao CC, Huang WH, Hsu YH, Hsu JC, Yen HC, Lin CL: Prognostic factors of spontaneous intracerebral haemorrhage in haemodialysis patients and predictors of 30-day mortality. Intern Med J 2008, 38(7):568-574

4. Pai MF, Hsu SP, Peng YS, Hung KY, Tsai TJ: Hemorrhagic stroke in chronic dialysis patients. Ren Fail 2004, 26(2):165-170.

5. Chang SF, Su CL, Chen ZY, Hung TP: Stroke incidence in Ilan, Taiwan. J Formos Med Assoc 1995, 94(1-2):30-36.

6. Su BG, Tsai KL, Yeh SH, Ho YY, Liu SY, Rivers PA: Risk factor and cost accounting analysis for dialysis patients in Taiwan. Health Serv Manage Res 2010, 23(2):84-93.

7. Chen HA, Wang JJ, Chou CT, Chien CC, Chu CC, Sheu MJ, Lin YJ, Chen PC, Chen $\mathrm{CH}$ : Predictors of long-term mortality in patients with and without systemic lupus erythematosus on maintenance dialysis: a comparative study. J Rheumatol 2011, 38(11):2390-2394.

8. Yoo DJ, Agodoa L, Yuan CM, Abbott KC, Nee R: Risk of intracranial hemorrhage associated with autosomal dominant polycystic kidney disease in patients with end stage renal disease. BMC Nephrol 2014 15(39):1-8.

9. Seliger SL, Gillen DL, Tirschwell D, Wasse H, Kestenbaum BR, Stehman-Breen CO: Risk factors for incident stroke among patients with end-stage renal disease. J Am Soc Nephrol 2003, 14(10):2623-2631.

10. Broderick JP, Adams HP Jr, Barsan W, Feinberg W, Feldmann E, Grotta J, Kase C, Krieger D, Mayberg M, Tilley B, Zabramski JM, Zuccarello M: Guidelines for the management of spontaneous intracerebral hemorrhage: a statement for healthcare professionals from a special writing group of the Stroke Council, American Heart Association. Stroke 1999, 30(4):905-915.

11. Tetri S, Juvela S, Saloheimo P, Pyhtinen J, Hillbom M: Hypertension and diabetes as predictors of early death after spontaneous intracerebral hemorrhage. J Neurosurg 2009, 110(3):411-417.

12. Arboix A, Massons J, García-Eroles L, Oliveres M, Targa C: Diabetes is an independent risk factor for in-hospital mortality from acute spontaneous intracerebral hemorrhage. Diabetes Care 2000, 23(10):1527-1532.

13. Lee TH, Ryu SJ, Chen ST: The prognostic value of blood glucose in patients with acute stroke. J Formos Med Assoc 1991, 90(5):465-470

14. Franke $\mathrm{CL}$, van Swieten JC, Algra A, van Gijn J: Prognostic factors in patients with intracerebral haematoma. J Neurol Neurosurg Psychiatry 1992, 55(8):653-657.

15. Hanel RA, Xavier AR, Mohammad Y, Kirmani JF, Yahia AM, Qureshi Al: Outcome following intracerebral hemorrhage and subarachnoid hemorrhage. Neurol Res 2002, 24(Suppl 1):S58-S62.

16. Brott $T$, Thalinger $K$, Hertzberg $V$ : Hypertension as a risk factor for spontaneous intracerebral hemorrhage. Stroke 1986, 17(6):1078-1083.

17. Okada H, Horibe H, Yoshiyuki O, Hayakawa N, Aoki N: A prospective study of cerebrovascular disease in Japanese rural communities, Akabane and Asahi. part 1: evaluation of risk factors in the occurrence of cerebral hemorrhage and thrombosis. Stroke 1976, 7(6):599-607.

18. Yang $Y$, Rosenberg GA: Blood-brain barrier breakdown in acute and chronic cerebrovascular disease. Stroke 2011, 42(11):3323-3328.

19. Liu J, Jin X, Liu KJ, Liu W: Matrix metalloproteinase-2-mediated occludin degradation and caveolin-1-mediated claudin-5 redistribution contribute to blood-brain barrier damage in early ischemic stroke stage. J Neurosci 2012, 32(9):3044-3057.

20. Rosell A, Ortega-Aznar A, Alvarez-Sabín J, Fernández-Cadenas I, Ribó M Molina CA, Lo EH, Montaner J: Increased brain expression of matrix metalloproteinase- 9 after ischemic and hemorrhagic human stroke. Stroke 2006, 37(6):1399-1406

21. Clark AW, Krekoski CA, Bou SS, Chapman KR, Edwards DR: Increased gelatinase A (MMP-2) and gelatinase B (MMP-9) activities in human brain after focal ischemia. Neurosci Lett 1997, 238(1-2):53-56.

22. Kim KR, Kim YZ: Clinical comparison of 30-day mortalities and 6-month functional recoveries after spontaneous intracerebral hemorrhage in patients with or without end-stage renal disease. J Korean Neurosurg Soc 2013, 54(3):164-174

23. Iseki K, Fukiyama K, Okawa Dialysis Study (OKIDS) Group: Clinical demographics and long-term prognosis after stroke in patients on chronic haemodialysis. the Okinawa Dialysis Study (OKIDS) Group. Nephrol Dial Transplant 2000, 15(11):1808-1813.

24. Chan $\mathrm{CL}$, Ting HW, Huang HT: The incidence, hospital expenditure, and, 30 day and 1 year mortality rates of spontaneous intracerebral hemorrhage in Taiwan. J Clin Neurosci 2014, 21(1):91-94.

25. Cardona-Gómez GP, Mendez P, DonCarlos LL, Azcoitia I, Garcia-Segura LM: Interactions of estrogen and insulin-like growth factor-I in the brain: molecular mechanisms and functional implications. J Steroid Biochem Mol Biol 2002, 83(1-5):211-217.

26. Roof RL, Hall ED: Gender differences in acute CNS trauma and stroke: neuroprotective effects of estrogen and progesterone. J Neurotrauma 2000, 17(5):367-388.

27. Zia E, Engström G, Svensson PJ, Norrving B, Pessah-Rasmussen $H$ : Three-year survival and stroke recurrence rates in patients with primary intracerebral hemorrhage. Stroke 2009, 40(11):3567-3573.

28. Olsen TS, Dehlendorff C, Andersen KK: Sex-related time-dependent variations in post-stroke survival-evidence of a female stroke survival advantage. Neuroepidemiology 2007, 29(3-4):218-225.

29. Krane NK: Intracranial pressure measurement in a patient undergoing hemodialysis and peritoneal dialysis. Am J Kidney Dis 1989, 13(4):336-339.

30. Yorioka N, Oda H, Ogawa T, Taniguchi Y, Kushihata S, Takemasa A, Usui K, Shigemoto K, Harada S, Yamakido M: Continuous ambulatory peritoneal dialysis is superior to hemodialysis in chronic dialysis patients with cerebral hemorrhage. Nephron 1994, 67(3):365-366.

31. Gondo G, Fujitsu K, Kuwabara T, Mochimatsu Y, Ishiwata Y, Oda H, Takagi N, Yamashita T, Fujino H, Kim I, Nakajina F: Comparison of five modes of dialysis in neurosurgical patients with renal failure. Neurol Med Chir (Tokyo) 1989, 29(12):1125-1131.

doi:10.1186/1471-2369-15-186

Cite this article as: Lin et al:: The impact of comorbidity on survival after hemorrhagic stroke among dialysis patients: a nationwide populationbased study. BMC Nephrology 2014 15:186.

\section{Submit your next manuscript to BioMed Central and take full advantage of:}

- Convenient online submission

- Thorough peer review

- No space constraints or color figure charges

- Immediate publication on acceptance

- Inclusion in PubMed, CAS, Scopus and Google Scholar

- Research which is freely available for redistribution 